\title{
A Fast Handwritten Digit Recognition Algorithm Based on Improved SVM
}

\author{
Qiong Li ${ }^{\text {a }}$, Li Chen ${ }^{\mathrm{b}}$
}

School of Computer Science and Technology, Hankou University, Wuhan 430212, China.

a813616@qq.com, b4036091@qq.com

Keywords: handwritten digit recognition, Support Vector Machine, kernel parameter, Separability Measure

\begin{abstract}
Handwritten digit recognition is of great value for application in the field of Image Processing and Pattern Recognition. For ensuring better recognition accuracy and speeding up classification process, this paper proposes a fast handwritten digit recognition method based on improved SVM. The new method uses the Separability Measure (SM) between classes in a high dimensional feature space to determine the best kernel parameters, it can fast train SVM classifiers to recognize handwritten digits. The computation of Separability Measure is a simple iterative process, thus the time required for computing SM is far less than that for training SVM classifiers in traditional parameter optimization methods. Therefore, the time for kernel parameters selection will be reduced greatly, the training process will be speeded up accordingly, and the recognition speed will be improved finally. Our experiments in the MNIST database demonstrate that the improved algorithm is feasible and effective.
\end{abstract}

\section{Introduction}

Handwritten digits recognition is a branch of Optical Character Recognition (OCR), its goal is to make computer automatically recognize handwritten Arabic numerals written on paper [1]. At present, handwritten digits recognition technology is widely applied in the fields of zip code, financial statements, statistical reports and bank notes, etc. It is a hot research topic of Image Processing and Pattern Recognition. Traditional handwritten digits recognition technologies, such as artificial classification, neural network and decision tree, they generally have the problems of slow recognition speed and low recognition accuracy. Thus, this paper proposed a fast handwritten digit recognition algorithm based on Support Vector Machine (SVM) [2]. And then we do experiments on MNIST dataset, the experimental results show that the proposed method can speed up SVM training process, improves recognition speed of handwritten digits and obtains better recognition accuracy.

\section{Recognition Principle of Handwritten Digits}

Handwritten digit recognition is an interdisciplinary complex problem [3]. It concerns many fields, such as Image Processing, Pattern Recognition and Machine Learning, etc. Its recognition system usually includes three parts: image preprocessing, feature extraction and classification recognition, as shown in Figure 1.

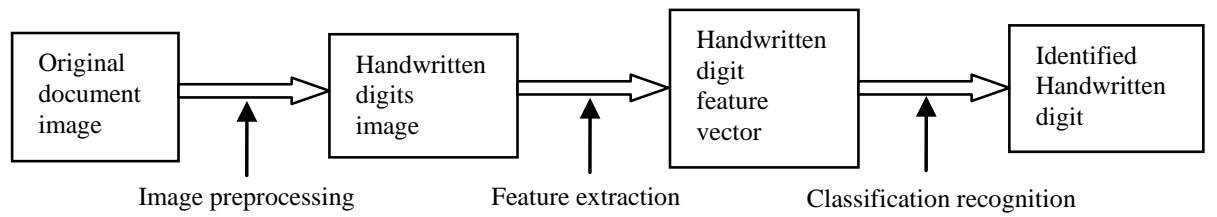

Fig. 1 Recognition principle of handwritten digits

Image Preprocessing. The first step of handwritten digits recognition is image preprocessing. Usually, the identifying handwritten digit images which are obtained by scanning have some noises. Because the scanning resolution is different, the image quality is also different. In addition, we also needs to split handwritten digits included document images correctly. After segmenting, the digits' 
size and font are usually different, thus, we need to normalize them. So image preprocessing mainly solves: image binarization, image smoothing, character segmentation, normalization, etc.

Feature Extraction. After image preprocessing, we use feature extraction algorithm to extract essential properties of digit image for recognizing. Generally, handwritten digital features include: statistical characteristic and structural characteristic. Statistical characteristic can be obtained by using mathematical transformation methods to process binary or grey scale image. And Structural characteristic mainly describe geometric structure of handwritten digits, it focuses on properties that can reflect digit's structure. In practical applications, we usually combine various feature extraction methods for obtaining the optimal feature vector.

Classification Recognition. Classification recognition is to use trained classifier to recognize handwritten digits after feature extraction. Classifier recognition principle is that defining a group of curved surfaces or hyper surfaces in feature space, and use them to divide feature space into different areas for achieving classification. At present, the commonly used classifiers include: classifier based on distance, neural network classifier and SVM classifier.

SVM classifier is to select a group of feature subsets called support vector to recognize. It makes the linear classification for support vector sets equal to segmentation for the whole data set, so as to achieve lower computation complexity and ensure the better classification accuracy [2]. Thus, we choose SVM classifier to recognize handwritten digits. Furthermore, in order to speed up training process, improve classification speed and obtain the better recognition accuracy, we use Separability Measure (SM) of each class in feature space to determine the optimal kernel parameters.

\section{A Fast Handwritten Digit Recognition Algorithm Based on Improved SVM}

Handwritten digits recognition is a multi-class classification problem(10 categories), but SVM was originally designed for binary classification. Thus, we need to extend it for multi-class classification. Currently, there are two types of approaches for multi-class SVM [4]. One is by constructing and combining several binary classifiers while the other is by directly considering all data in one optimization formulation. Because the latter one is time consuming, we usually use the first one. In general, there are four approaches for constructing SVM multi-class classifiers: 1-a-r (1-against-rest), 1-a-1 (1-against-1), DAG-SVM and Binary tree SVM. We use 1-a-1 to solve the handwritten digits classification. 1-a-1 method need to construct $k(k-1) / 2(k(k>=2)$ is the number of classes) SVM classifiers where each one is trained on data from two classes. And then we use each SVM classifier trained to recognize an unknown digit sample. Finally, we use "voting" to determine its category.

SVM is a machine learning method based on kernel. Therefore, we need to determine the kernel and its parameters in SVM [5]. This will directly affect the performance of SVM classifiers [6]. Among three common kernels, Gaussian kernel has been shown to give competitive learning ability, thus, we choose Gaussian kernel as SVM Mercer kernel. The next is to how to determine kernel parameters. Currently, Grid Search is the commonly used method to optimize kernel parameters. It obtains a 2 dimension grid by estimating the variation of kernel parameters, and each node in the grid will be seen as a set of candidate parameters. Then it uses Cross Validation method to achieve according classification accuracy of each candidate parameter, and select the combination of parameters which has the best classification accuracy as the optimal kernel parameter. However, Grid Search method has the problem that optimization process is very slow, and the real-time processing cannot be realized yet.

In order to improve optimization speed, this paper uses the Separability Measure of each class in high dimensional feature space to determine the optimal kernel parameter. Due to not training corresponding SVM model, the proposed method could save training time, speeds up training process, and improves the handwritten digital recognition speed.

Given two handwritten digit instances in input space $x_{1},{ }^{x_{2}}$, where ${ }^{x_{1}}$ and $x_{2}$ are the feature vectors of handwritten digit instances, then we use the kernel function $\phi$ to map them into a higher 
dimensional Hilbert feature space, and get: $\phi\left(x_{1}\right), \phi\left(x_{2}\right)$. Thus, the dot product of input samples in Hilbert feature space should be expressed as:

$$
K\left(x_{1}, x_{2}\right)=\phi\left(x_{1}\right) \cdot \phi\left(x_{2}\right)
$$

Accordingly, the Euclidean distance between $x_{1}$ and $x_{2}$ in Hilbert space is:

$$
d^{H}\left(x_{1}, x_{2}\right)=\sqrt{K\left(x_{1}, x_{1}\right)-2 K\left(x_{1}, x_{2}\right)+K\left(x_{2}, x_{2}\right)}
$$

Its class center $m_{\phi}$ in feature space is:

$$
m_{\phi}=\frac{1}{n} \sum_{i=1}^{n} \phi\left(x_{i}\right)
$$

Here, $n$ is the total number of instances.

Given two groups of training samples: $\left\{x_{1}, x_{2}, \cdots, x_{n 1}\right\}$ and $\left\{y_{1}, y_{2}, \cdots, y_{n 2}\right\}$, then we use $\phi$ to map them into a higher dimensional feature space, and we will get two class centers: $m_{\phi}$ and $m_{\phi}^{\prime}$. Thus, the Euclidean distance between $m_{\phi}$ and $m_{\phi}^{\prime}$ in Hilbert space is:

$$
d^{H}\left(m_{\phi}, m_{\phi}^{\prime}\right)=\sqrt{\frac{1}{n_{1}^{2}} \sum_{i=1}^{n_{1}} \sum_{j=1}^{n_{1}} K\left(x_{i}, x_{j}\right)-\frac{2}{n_{1} n_{2}} \sum_{i=1}^{n_{1}} \sum_{j=1}^{n_{2}} K\left(x_{i}, y_{j}\right)+\frac{1}{n_{2}^{2}} \sum_{i=1}^{n_{2}} \sum_{j=1}^{n_{2}} K\left(y_{i}, y_{j}\right)}
$$

Accordingly, the Euclidean distance between training instance $x$ and its class center $m_{\phi}$ in Hilbert space is:

$$
d^{H}\left(x, m_{\phi}\right)=\sqrt{K(x, x)-\frac{2}{n} \sum_{i=1}^{n} K\left(x, x_{i}\right)+\frac{1}{n^{2}} \sum_{i=1}^{n} \sum_{j=1}^{n} K\left(x_{i}, x_{j}\right)}
$$

Thus, we define Separability Measure (SM) between class $i$ and class $j$ in feature space as:

$$
s m_{i j}^{H}=\frac{d^{H}\left(m_{\phi}^{i}, m_{\phi}^{j}\right)}{\sigma_{i}^{H}+\sigma_{j}^{H}}
$$

Here, $\sigma^{H}=\max d^{H}\left(x_{i}, m_{\phi}\right)$ is the maximum of distances between each point and its class center, representing the compactness of one class.

The fast kernel parameters optimization algorithm (given training set is $L$, validation set is $M$, testing set is $N$, and $K(K \geq 2)$ is the total number of handwritten digit categories):

Step1: Predefine a set of candidate kernel parameters. For each candidate parameter, compute the defined Separability Measure $s m^{H}$ on training set $L$;

Step2: Sort the $s m^{H}$ in ascending order, the parameter which has the maximum of $s m^{H}$ is the optimal kernel parameter;

Step3: Predefine a set of penalty factors. For each parameter combination ( $\left.C, \sigma_{\text {Best }}\right)$, train the corresponding SVM classifiers;

Step4: Use all SVM classifiers obtained in Step3 to verify $M$. The combination which has the maximum of validation accuracy is the optimal kernel parameter combination $\left(C_{\text {Best }}, \sigma_{\text {Best }}\right)$;

Step5: Use the optimal SVM classifier obtained in Step4 to recognize testing set $N$.

\section{Experiments and Analysis}

In our experiment, all handwritten digit instances considered are from MNIST. MNIST is a database provided by NIST, and it is specially used for handwritten digit recognition (download link: http://yann.lecun.com/exdb/mnist/).

We randomly select 1000 handwritten digit instances as training samples, 600 instances as validation samples, and 400 instances as testing samples. The instance vector dimension is $20 * 20=400$. In the experiment, we use 5 -fold cross-validation to obtain validation accuracy, use SMO to train SVM classifiers, and use 1-a-1 method to solve handwritten digit multi-class classification problem. LIBSVM [7, 8] used in the experiment can be available at http://www.csie.ntu.edu.tw/. In addition, We use Gaussian kernel function and set the search scopes of kernel parameters in advance: $C \in\left[2^{-1}, 2^{0}, \cdots, 2^{7}\right], \sigma \in\left[2^{-15}, 2^{-14}, \cdots, 2^{0}\right]$. Finally, we compare 
performance by the proposed algorithm with that by the Grid Search method. The experimental results are shown in Table 1.

Table 1 Experimental results

\begin{tabular}{cccc}
\hline & $\begin{array}{c}\text { Kernel parameters } \\
(C, \sigma)\end{array}$ & Training time(s) & Recognition accuracy(\%) \\
\hline Grid Search & $\left(2^{3}, 2^{-14}\right)$ & 265 & 94.03 \\
The improved method & $\left(2^{2.5}, 2^{-12}\right)$ & 74 & 94.21 \\
\hline
\end{tabular}

The experimental results indicate that, compared to the Grid Search method, the improved kernel parameters optimization algorithm can obtain better classification accuracy, saves training time efficiently, and improves handwritten digit recognition speed accordingly.

\section{Conclusions}

In this paper, we introduce the principle of handwritten digit recognition firstly, and then we propose a fast handwritten digit recognition algorithm based on improved SVM. The proposed method uses the Separability Measure of each class in feature space to determine the optimal kernel parameters. Compared to the traditional Grid Search method, the computation of Separability Measure is very simple and doesn't need iteration, therefore, the new method could effectively save training time, enjoys much faster training, increases handwritten digits recognition speed, and achieves better classification precision. Experiments on MNIST demonstrate that the improved algorithm is feasible and effective. The next work is how to preset reasonable parameter search scope so as to shorten kernel parameters optimization time further.

\section{Acknowledgements}

This study is supported by the Science and Technology Research Planning Foundation of Education Bureau of Hubei Province of China (No. B20128103).

\section{References}

[1] Z.Q. Bian, X.G. Zhang: Pattern Recognition (Tsinghua University Press, China 2000) (In Chinese)

[2] V.N. Vapnik: Statistical Learning Theory (Electronic Industry Press, China 2004)

[3] A.G. Zippo, G. Gelsomino, S. Nencini and G.E.M. Biella: Proc. 22nd Italian Workshop on Neural Nets (Vietri sul Mare, Salerno, Italy, May 17-19, 2012). Vol. 19 (2013), p.189-200.

[4] D. Abdelhamid, T.A. Abdelmalik: International Journal of Computer Science Issues, Vol. 8 (2011) No.5, p.544-550.

[5] J.L. Yin, Y.L. Zhu: Electrical Measurement \& Instrumentation, Vol. 49 (2012) No.557, p.11-16. (In Chinese)

[6] J.F. Wang, L. Zhang, G.X. Chen and X.W. He: Applied Science and Technology, Vol. 39 (2012) No.3, p.28-31. (In Chinese)

[7] Information on http://www.csie.ntu.edu.tw/ cjlin/papers/guide/guide.pdf

[8] Information on http://www.csie.ntu.edu.tw/ cjlin/papers/libsvm.pdf 